04

\title{
О форме канала тлеющего разряда
}

\author{
() А.Л. Дмитриев, Е.М. Никущенко
}

Университет ИТМО,

197101 Санкт-Петербург, Россия

e-mail: alex@dmitriyev.ru

(Поступило в Редакцию 29 сентября 2016 г.)

Приведены примеры формы канала тлеющего разряда в переменном токе частотой $50 \mathrm{~Hz}$, в воздухе при давлении $0.1 \mathrm{~atm}$ и эффективном значении силы тока 30-70 mA. Показано, что при указанных давлении и силе тока тепловая конвекция в вакуумной камере и плавучесть (сила Архимеда) не являются главными причинами своеобразной параболической формы тлеющего разряда.

DOI: 10.21883/JTF.2017.05.44460.2049

Изучению свойств тлеющего разряда посвящено множество публикаций, при этом большинство описанных в литературе экспериментов выполнялось в разрядных трубках или в специальных камерах [1-3]. Весьма подробно форма тлеющего разряда при постоянном токе исследовалась в 30-е годы прошлого века [4,5]. В научной литературе утвердилось мнение, что в отсутствии сильных внешних электрического и магнитного полей главными причинами характерной дугообразной формы протяженного разряда в атмосфере являются воздушная конвекция и архимедова сила выталкивания нагретого токового канала. Описываемый эксперимент выполнен с целью оценки степени влияния указанных факторов в тлеющем разряде переменного тока в воздухе при давлении $0.1 \mathrm{~atm}$ и эффективном значении силы тока в диапазоне 30-70 mA. Частота тока $50 \mathrm{~Hz}$, напряжение на дюралевых электродах в пределах $0.6-1.0 \mathrm{kV}$, расстояние между электродами $45 \mathrm{~mm}$.

Примеры фото канала тлеющего разряда показаны на рис. 1.

При времени экспозиции $33 \mathrm{~ms}$ (рис. 1, $a, b)$ фотокамерой фиксируются два пространственно разделенных разряда, каждому из которых соответствует определенное, длительностью $10 \mathrm{~ms}$ (половина периода колебаний), направление тока. Относительный сдвиг траекторий разрядов на этих рисунках обусловлен влиянием силы Лоренца в лабораторном магнитном поле Земли, тангенциальная компонента которого направлена поперек разряда.

За время экспозиции $2.5 \mathrm{~ms}$ (рис. 1,c. фиксируется тлеющий разряд при одном направлении тока, здесь хорошо различимы катодное темное пространство и пространство Астона. Рис. 1, с показывает, что формирование тлеющего разряда происходит за время, по крайней мере, меньшее или около полупериода колебаний тока.

Правильная, близкая к параболе траектория тлеющего разряда на рис. 1, по-видимому, формируется вследствие восходящего конвекционного потока воздуха в вакуумной разрядной камере либо в результате действия на плазменный канал силы плавучести. Примечательно, что при вращении разрядной камеры на угол $90^{\circ}$ вокруг горизонтальной оси, направление которой совпадает с направлением электродов, плоскость параболы тлеющего разряда остается вертикальной. Для устранения возможного влияния на исследуемый разряд внешнего

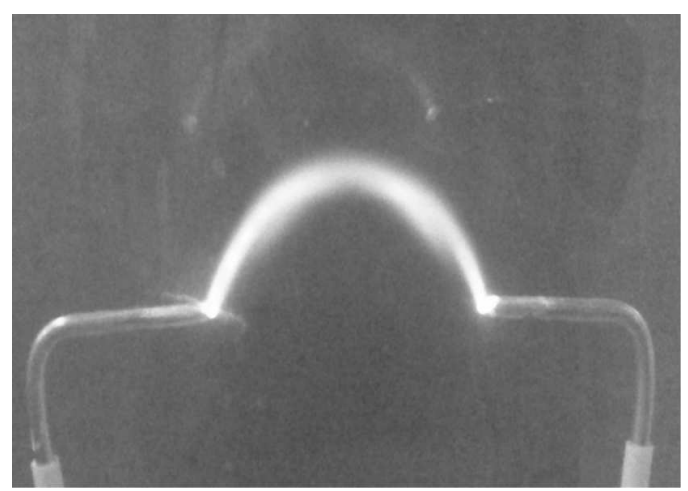

$a$

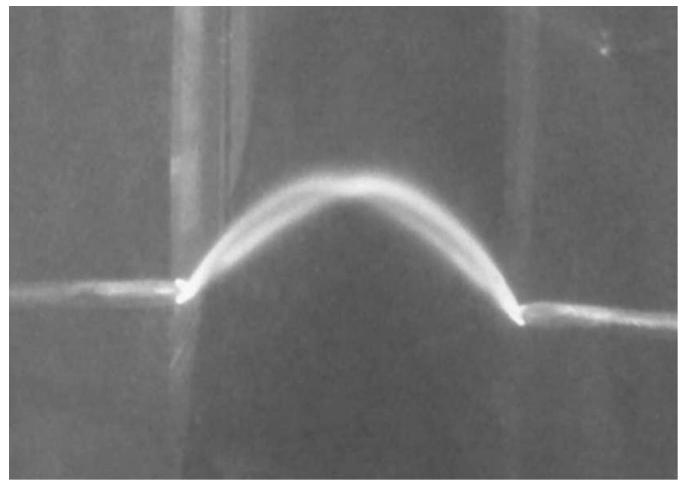

$b$

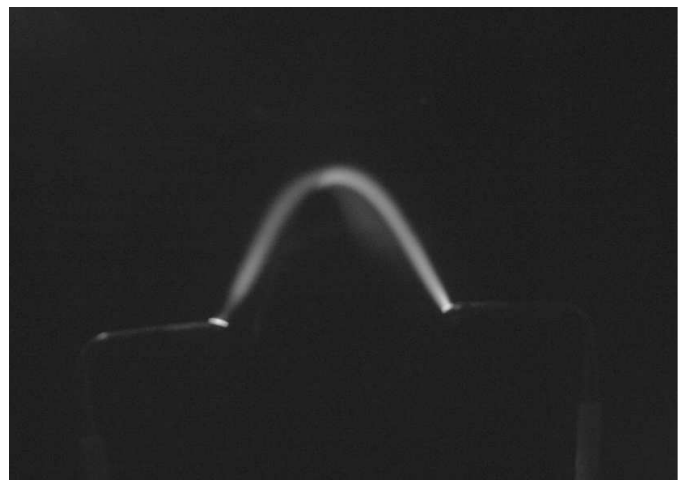

c

Рис. 1. Фото канала тлеющего разряда. Экспозиция: $a, b-$ $33 \mathrm{~ms}, c-2.5 \mathrm{~ms}$. 


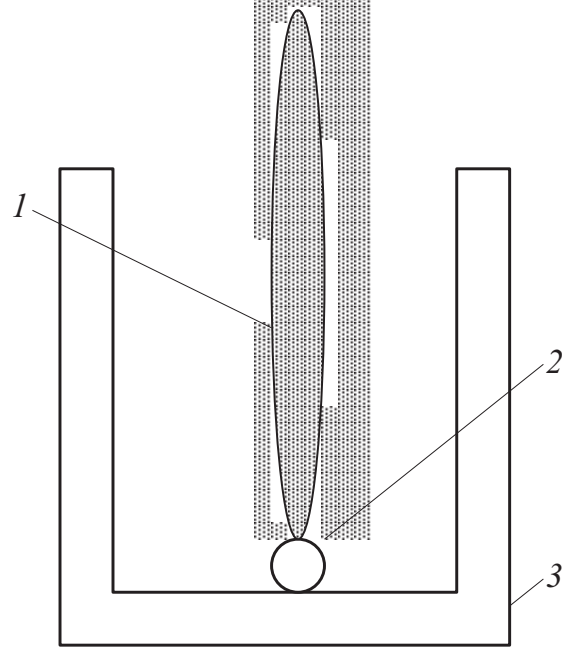

Рис. 2. Взаимное расположение канала тлеющего разряда 1 , электрода 2 , и ограничителя восходящего потока воздуха 3.

(лабораторного) электрического поля разрядная камера экранировалась стальными щитами толщиной $0.5 \mathrm{~mm}$ тем не менее форма, ориентация и размеры канала тлеющего разряда практически не изменялись.

Для ослабления интенсивности восходящего конвекционного потока исследуемый разряд помещался в открытый прямоугольный ящик размерами $25 \times 25 \times 125 \mathrm{~mm}$, изготовленный из текстолита, при этом дно ящика находилось в непосредственной близости от электродов и разряда; поперечные сечения ограничителя, электрода и плазменного канала схематически показаны на рис. 2. Характерный результат описываемых экспериментов состоит в том, что при постоянных токе разряда и давлении воздуха в камере форма и высота параболического канала тлеющего разряда не зависели от положения ящика - ограничителя восходящего конвекционного потока.

Полагая, что главной причиной дугообразной формы тлеющего разряда является архимедова сила выталкивания, оценим время „всплывания“ горячего канала такого разряда. Температурные и газодинамические процессы, сопровождающие формирование электрического разряда в атмосфере, весьма сложны. Порядок величины времени всплывания канала тлеющего разряда приближенно можно оценить на основе следующих элементарных выкладок.

Электрический разряд представим в виде цепочки сфер радиуса $R$, заполненных плазмой с плотностью $\rho_{p}$. Сила выталкивания такой цепочки уравновешивается ее весом и силой трения, для оценки величины которой воспользуемся известной формулой Стокса:

$$
\frac{4}{3} \pi R^{3}\left(\rho_{a}-\rho_{p}\right) g=6 \pi v R u,
$$

где $\rho_{a}-$ плотность воздуха, $g-$ ускорение силы тяжести, $v-$ вязкость воздуха, $u-$ средняя скорость всплывания цепочки. Полагая $\rho_{a} \gg \rho_{p}$, время $t$ всплыва- ния цепочки на высоту $h, t=h / u$ равно

$$
t=\frac{9}{2} \frac{v h}{g R^{2}\left(\rho_{a}-\rho_{p}\right)}>4.5 \frac{v h}{g R^{2} \rho_{a}} .
$$

Подставляя в правую часть (2) численные значения, близко соответствующие условиям описываемого эксперимента $\left(h \approx 1.6 \mathrm{~cm}, R \approx 1 \mathrm{~mm}, \rho_{a} \approx 0.1 \cdot 1.3 \mathrm{~kg} / \mathrm{m}^{3}\right.$, $v \sim 2 \cdot 10^{-5} \mathrm{~kg} / \mathrm{m} \cdot \mathrm{s}, g \approx 9.8 \mathrm{~m} / \mathrm{s}^{2}$, здесь $0.1 \mathrm{~atm}$ соответствует $0.13 \mathrm{~kg} / \mathrm{m}^{3}$, а для вязкости, величина которой увеличивается с температурой, выбрано указанное минимальное значение [6]), получим в итоге $t>1 s$. Очевидно, приведенная величина $t$ сушественно превышает время установления дугообразного тлеющего разряда (около $10 \mathrm{~ms}$ ). Следовательно, плавучесть (сила Архимеда) также не является главной причиной своеобразной формы канала тлеющего разряда.

Итак, описанный эксперимент показывает, что при указанных выше давлении и силе тока тепловая конвекция в вакуумной камере и плавучесть (сила Архимеда) не являются причинами параболической (дуговой) формы канала тлеющего разряда. Особенности динамики и структуры протяженного тлеющего разряда переменного тока должны быть предметом тщательных специальных исследований.

\section{Список литературы}

[1] Райзер Ю.П. Физика газового разряда. М.: ГРФМЛ, 1987. $592 \mathrm{c.}$

[2] Ховатсон А.М. Введение в теорию газового разряда. М.: Атомиздат, 1980. $182 \mathrm{c}$

[3] Биттенкорт Ж.А. Основы физики плазмы. М.: ФИЗМАТЛИТ, 2009. 584 c.

[4] Энгель А. Ионизованные газы. М.: ГИФМЛ, 1959. 332 с.

[5] Steenbeck M. // Phys. Zeitschrift. 1937. Vol. 38, N 23. P. 10191021.

[6] Бабичев А.П., Бабушкина Н.А., БратковскийА.М. и др. Физические величины. Справочник. М.: Энергоатомиздат, 1991. $1232 \mathrm{c}$. 